\title{
Characteristics of a prototype matrix of Silicon PhotoMultipliers (SiPM)
}

\section{N. Dinu,,${ }^{a, 1,2}$ P. Barrillon, ${ }^{a}$ C. Bazin, ${ }^{a}$ N. Belcari, ${ }^{b, c}$ M.G. Bisogni, ${ }^{b, c}$ S. Bondil-Blin, ${ }^{a}$ M. Boscardin, ${ }^{d}$ V. Chaumat, ${ }^{a}$ G. Collazuol, ${ }^{e, c}$ C. De La Taille, ${ }^{a}$ A. Del Guerra, ${ }^{b, c}$ G. Llosá, ${ }^{b}$ S. Marcatili, ${ }^{b, c}$ M. Melchiorri, ${ }^{d}$ C. Piemonte,${ }^{d}$ V. Puill, ${ }^{a}$ A. Tarolli, ${ }^{d}$} J.F. Vagnucci ${ }^{a}$ and N. Zorzi ${ }^{d}$

\author{
${ }^{a}$ Laboratory of Linear Accelerator (LAL), IN2P3-CNRS, \\ 91898 Orsay, France \\ ${ }^{b}$ Università di Pisa, Dipartimento di Fisica "E. Fermi", \\ 56127 Pisa, Italy \\ ${ }^{c}$ INFN, Sezione di Pisa, \\ 56127 Pisa, Italy \\ ${ }^{d}$ Fondazione Bruno Kessler (FBK-irst), \\ 38050 Trento , Italy \\ ${ }^{e}$ Scuola Normale Superiore (SNS), \\ 56127 Pisa, Italy \\ E-mail: dinu@lal.in2p3.fr
}

ABSTRACT: This work reports on the electrical (static and dynamic) as well as on the optical characteristics of a prototype matrix of Silicon Photomultipliers (SiPM). The prototype matrix consists of $4 \times 4$ SiPM's on the same substrat fabricated at FBK-irst (Trento, Italy). Each SiPM of the matrix has an area of $1 \times 1 \mathrm{~mm}^{2}$ and it is composed of 625 microcells connected in parallel. Each microcell of the SiPM is a GM-APD ( $\mathrm{n}^{+} / \mathrm{p}$ junction on P+ substrate) with an area of $40 \times 40 \mu \mathrm{m}^{2}$ connected in series with its integrated polysilicon quenching resistance. The static characteristics as breakdown voltage, quenching resistance, post-breakdown dark current as well as the dynamic characteristics as gain and dark count rate have been analysed. The photon detection efficiency as a function of wavelength and operation voltage has been also estimated.

KEYWORDS: Photon detectors for UV, visible and IR photons (solid-state); Particle detectors

\footnotetext{
${ }^{1}$ Corresponding author

${ }^{2}$ On leave from Institute of Space Sciences, Bucharest, Romania
} 


\section{Contents}

1 Introduction 1

2 Experimental 2

2.1 Prototype matrix of SiPM's 2

2.2 Measurement set-ups 2

$\begin{array}{lll}2.2 .1 & \text { Electrical measurement set-up } & 2\end{array}$

2.2.2 Optical measurement set-up 3

3 Characteristics of the prototype matrix 4

3.1 Electrical characteristics 4

3.1.1 Static characteristics 4

3.1.2 Dynamic characteristics 4

$\begin{array}{lll}3.2 & \text { Optical characteristics } & 7\end{array}$

4 Conclusions $\quad 8$

\section{Introduction}

Efficient detectors for low-light-level detection and photon counting are today required in a large variety of fields including astroparticle physics, nuclear medicine and high-energy physics. The Silicon PhotoMultiplier [1-3] represents a good candidate for such applications since it presents important advantages like high speed (the rise time of the signal $\sim$ hundreds of ps), high internal gain $\left(\sim 10^{5}-10^{6}\right)$, low power consumption $(\mathrm{V}<100 \mathrm{~V}$, I $\sim$ tens of $\mu \mathrm{A})$, single photon counting resolution, insensitive to magnetic fields, stability and compactness. The detection area represents a limiting parameter of the SiPM, most of the devices produced up today having $1 \times 1 \mathrm{~mm}^{2}$ area. SiPM's of $3 \times 3 \mathrm{~mm}^{2}$ and $4 \times 4 \mathrm{~mm}^{2}$ have been also produced, but they are limited to be used in the mentioned applications since the noise level scales with the area of the device [4, 5]. Therefore, 2D matrices of $1 \times 1 \mathrm{~mm}^{2}$ SiPM devices on the same substrat are suitable. Such devices increase the detection area maintaining on each read-out channel a noise level corresponding to a SiPM of $1 \mathrm{~mm}^{2}$ and gives in the same time 2D position information with a spatial resolution of $1 \mathrm{~mm}^{2}$.

2D prototype matrices of SiPM's have been developed at Fondazione Bruno Kessler (FBK-irst, Trento, Italy). Based on a scientific collaboration started during 2007 in between the Laboratory of Linear Accelerator (LAL, Orsay, France), FBK-irst and INFN-University of Pisa (Pisa, Italy) the characterization of one prototype matrix has been performed at LAL. The group of the Pisa University is mainly focused on the application of the system composed on the matrix of SiPM's and the multi-channel readout electronics developed at LAL for medical applications [6].

This paper presents the electrical (static and dynamic) as well as the optical characteristics of mentioned prototype matrix. In particular, static characteristics as breakdown voltage $\left(\mathrm{V}_{B D}\right)$, 


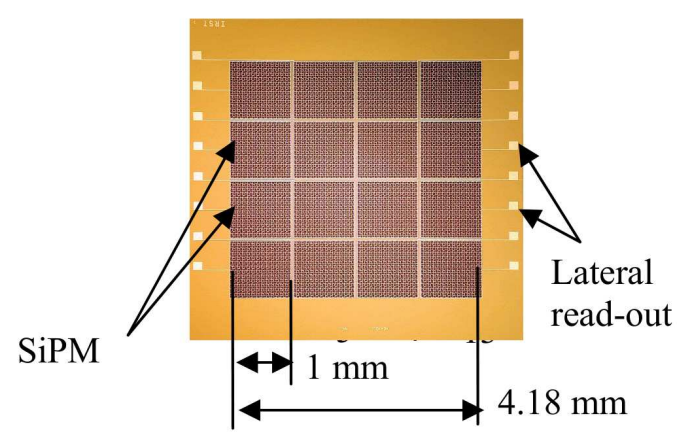

Figure 1. Layout of the prototype matrix of $4 \times 4$ SiPM'.

quenching resistance $\left(\mathrm{R}_{q}\right)$, post-breakdown dark current $\left(\mathrm{I}_{\text {post }-B D}\right)$ are analyzed as well as dynamic characteristics as gain $(\mathrm{G})$ and dark count rate (DCR). The photon detection efficiency (PDE) of this device as a function of wavelength $(\lambda)$ and operation voltage $\left(\mathrm{V}_{\text {bias }}\right)$ has been also estimated.

\section{Experimental}

\subsection{Prototype matrix of SiPM's}

The 2D prototype matrix of the SiPM's presented in this paper is composed of $4 \times 4$ SiPM's on the same substrate as shown in the figure 1 .

Each SiPM device, representing one read-out element of the matrix, has an area of $1 \times 1 \mathrm{~mm}^{2}$ and it is composed of 625 microcells connected in parallel. Each microcell of the SiPM has an area of $40 \times 40 \mu \mathrm{m}^{2}$ and it is represented by a GM-APD $\left(\mathrm{n}^{+} / \mathrm{p}\right.$ junction on $\mathrm{p}^{+}$substrate) connected in series with its integrated polysilicon resistance $\mathrm{R}_{q}$. The total fill factor of the matrix is $\sim 30-35 \%$, calculated as a product of the matrix fill factor $(\sim 92 \%)$ and the SiPM fill factor $(\sim 35-40 \%)$. The read-out of each SiPM in the matrix is performed lateraly, as shown in figure 1.

\subsection{Measurement set-ups}

The characterization of the matrix consists on the electrical test (static and dynamic) as well as on the optical test of each of the 16 SiPM elements. For these tests, different set-ups have been used as presented in the following.

\subsubsection{Electrical measurement set-up}

The electrical measurement set-up used for static tests (figure 2) consists on the connection of each SiPM of the matrix to Keithley 2612 source-meter $\left(\mathrm{V}_{\max }=200 \mathrm{~V}\right.$, $\mathrm{I}_{\text {sensibility }} \sim 2 \mathrm{pA}$, connections through triaxial cables $\mathrm{R}_{\text {isolation }} \sim 10 \mathrm{~T} \Omega$ ).

The electrical measurement set-up used for dynamic tests has two configurations. The first one (figure 3) consists on progressively connection of each SiPM of the matrix to a MITEQ AU-1332 single-channel wide-band $(0.01-500 \mathrm{MHz})$ voltage amplifier read-out by a Tektronix TDS 5054 $(500 \mathrm{MHz}, 5 \mathrm{GS} / \mathrm{s})$ digital oscilloscope. The amplifier presents a $50 \Omega$ input impedance, wich acts 


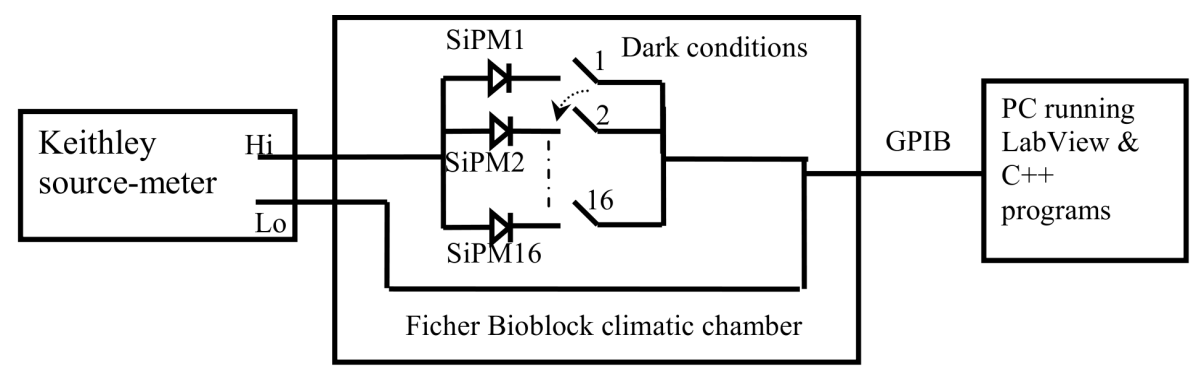

Figure 2. The electrical measurement set-up used for static tests.

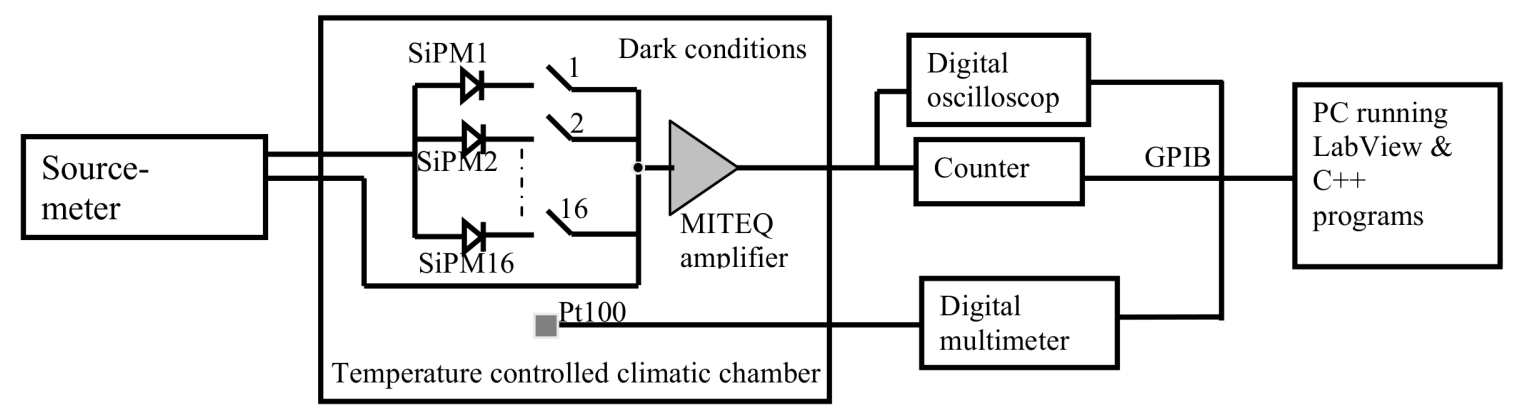

Figure 3. The first configuration of the electrical measurement set-up used for dynamic tests.

as a current-to-voltage converter followed by an amplifying stage having a gain of $45 \mathrm{~dB}$. LabView and $\mathrm{C}++$ programs have been developed for the automatic data acquisition and analysis of the SiPM parameters.

The second dynamic set-up (figure 4) includes a parallel connection of the 16 SiPM's of the matrix to the multi-channels read-out electronics called MAROC2 (Multi-Anod Read-Out Chip) read-out by a Lecroy digital oscilloscope. The MAROC2 has been designed at LAL to read-out the 64 channels of the MultiAnode Photomultiplier Tubes (MaPMT) for the ATLAS luminometer [7].

Based on the observation that a signal triggered by an absorbed photon and by a thermally generated carrier are identical, both dynamic tests (e.g. MITEQ amplifier or MAROC2 chip) have been performed in dark conditions.

A controlled temperature of both static and dynamic tests $\left(25 \pm 0.5^{\circ} \mathrm{C}\right)$ have been assured by Fisher Bioblock LMS A49401 climatic chamber.

\subsubsection{Optical measurement set-up}

The optical test has been performed using a halogen continuous light source $(100 \mathrm{~W})$ followed by a LOT ORIEL 77250 grating monochromator which separates the visible wavelengths (350$800 \mathrm{~nm}$ ) with an accuracy of $0.5 \mathrm{~nm}$. The incident flux, evaluated by two calibrated photodiodes (HPK S3590-18, UDT Instrument 221) was kept low $\left(\sim 10^{7} /\right.$ incident photons $\left./ \mathrm{s} / \mathrm{mm}^{2}\right)$ to avoid the SiPM and the acquisition system saturation. A precise positioning of the SiPM matrix and the calibrated diode in the light beam was assured by Polytec PI M413 tridimentional translation stage. The optical characteristics of each SiPM in the matrix have been evaluated using two data acquisition systems: the electrical set-up described in figure 2 (photocurrent technique) and the 


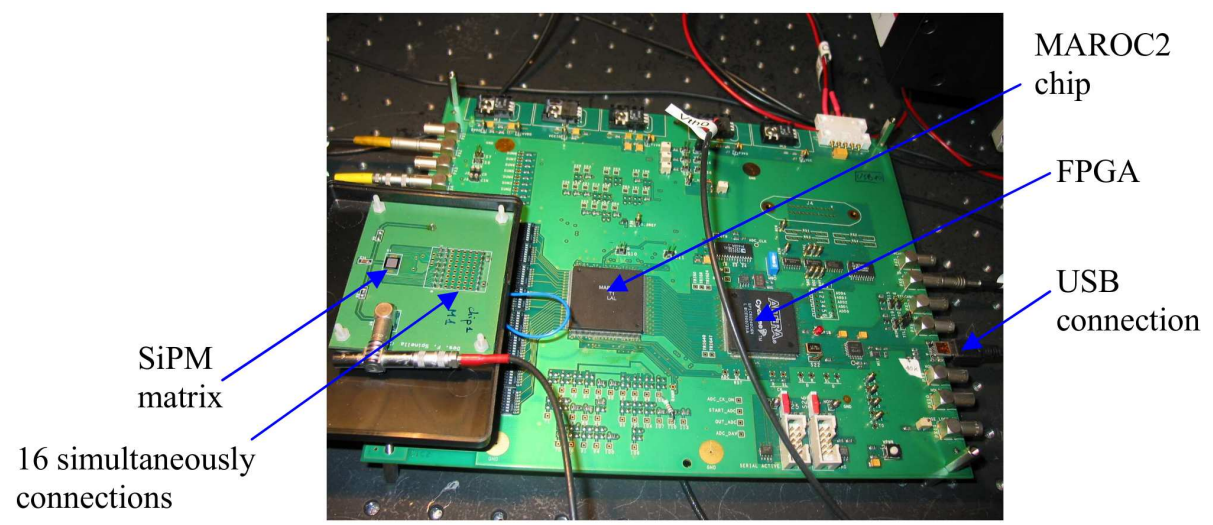

Figure 4. The second configuration of the electrical measurement set-up used for dynamic tests.

electrical set-up described in figure 3 (photon counting technique). Details about these techniques and the methods of PDE calculations will be given in the following section.

The optical test has been performed at room temperature $\left(25 \pm 1^{\circ} \mathrm{C}\right)$.

\section{Characteristics of the prototype matrix}

\subsection{Electrical characteristics}

\subsubsection{Static characteristics}

The static characteristics (reverse and forward IV plots) of the 16 SiPM's of the matrix are presented in figure $6 a, b$.

From the reverse IV plots, a mean value of $\mathrm{V}_{B D} \sim 30.5 \mathrm{~V}$ has been determined, with a very good uniformity over the 16 elements $(\sigma \sim 0.5 \%)$. The analysis of the $\mathrm{I}_{p o s t-B D}$ has been also performed, showing that $\mathrm{I}_{\text {post }-B D}$ lies in between two parabolic trends corresponding to the elements SiPM13 (lowest) and SiPM16 (highest). The mean value of the $\mathrm{I}_{\text {post }-B D}$ has been calculated, showing a value of $\sim 0.5 \mu \mathrm{A} @ \mathrm{~V}_{\text {bias }}=33 \mathrm{~V}$ (the overvoltage $\Delta \mathrm{V}=\mathrm{V}_{\text {bias }}-\mathrm{V}_{B D}=2.5 \mathrm{~V}$ ) with a dispersion of $\sim 12.5 \%$.

From the forward IV plots, a mean value of $\mathrm{R}_{q S i P M}$ of $\sim 500 \Omega$ has been found over the 16 elements of the matrix, with a very good uniformity $(\sigma \sim 1 \%)$. Considering that this represents the equivalent resistance of 625 pixels connected in parallel, a mean value of $\mathrm{R}_{\text {qpixel }} \sim 330 \mathrm{k} \Omega$ has been calculated.

\subsubsection{Dynamic characteristics}

The analysis of any SiPM of the matrix in dynamic conditions (using the set-up of MITEQ amplifier), revealed different types of signals [8] as shown in figure 7:

- signals coming from single pixels (e.g. primary pulses); they are generated by thermal carriers and represent the main source of the SiPM noise;

- signals following the primary pulses at short interval of time (e.g. afterpulses); they represent carriers trapped during one avalanche and when they released (during tens of ns), they trigger a new avalanche; 


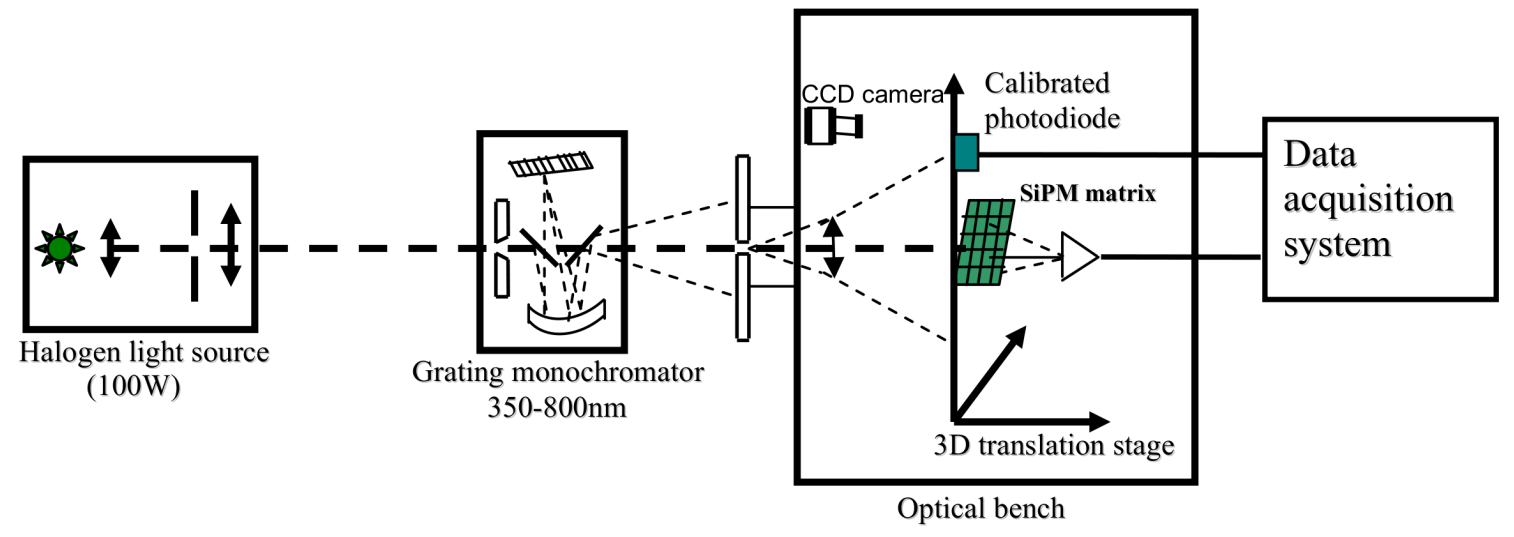

Figure 5. The optical measurement set-up.

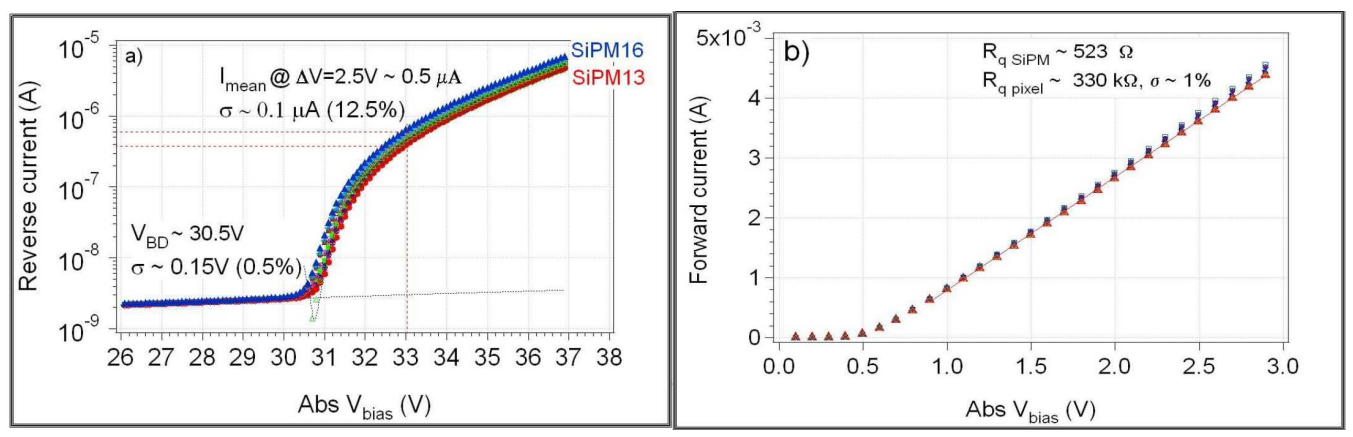

Figure 6. a) Reverse and b) forward IV characteristics of the $16 \mathrm{SiPM}$ elements of the matrix.

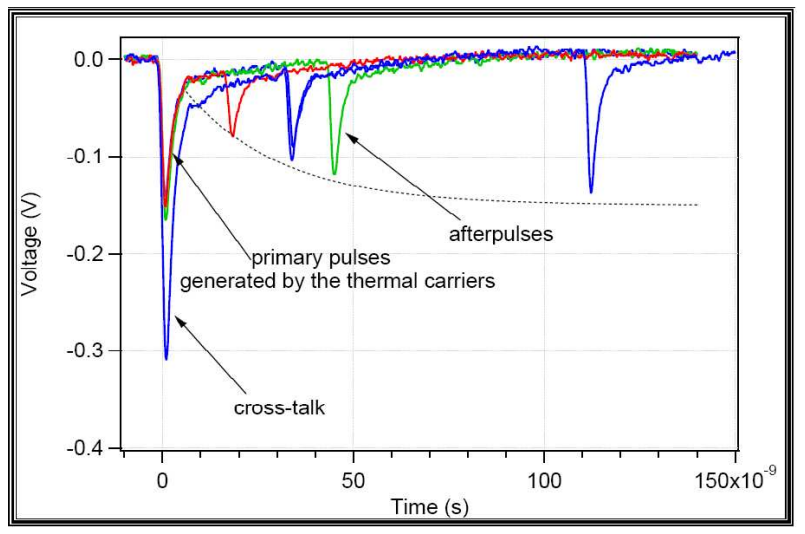

Figure 7. SiPM dark signals shape.

- signals coming from one or more neighbours pixels overlaid with the primary pulses (e.g. optical cross-talk); such signals are generated by photons created during the primary avalanche which can trigger avalanches in the adjacent pixels.

Counting all types of signals presented in figure 7, the dark count rate (DCR) can be determined. The DCR (counts/s) as a function of the $\mathrm{V}_{\text {bias }}$ is represented in figure 8 for the SiPM13 (the lowest $\mathrm{I}_{\text {post }-B D}$ ) and the SiPM16 (the highest $\mathrm{I}_{\text {post }-B D}$ ). The DCR of all the others SiPM's in the matrix are also represented, at $\mathrm{V}_{\text {bias }}$ of $33 \mathrm{~V}$. 


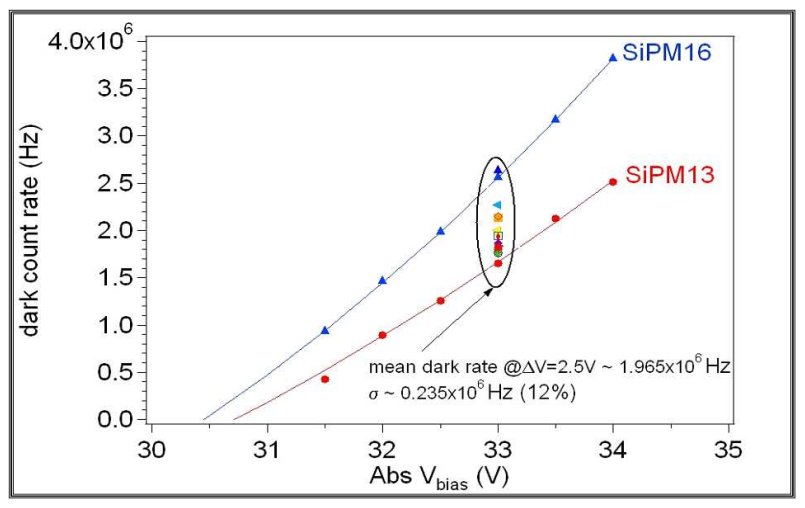

Figure 8. Dark count rate vs. the operation.

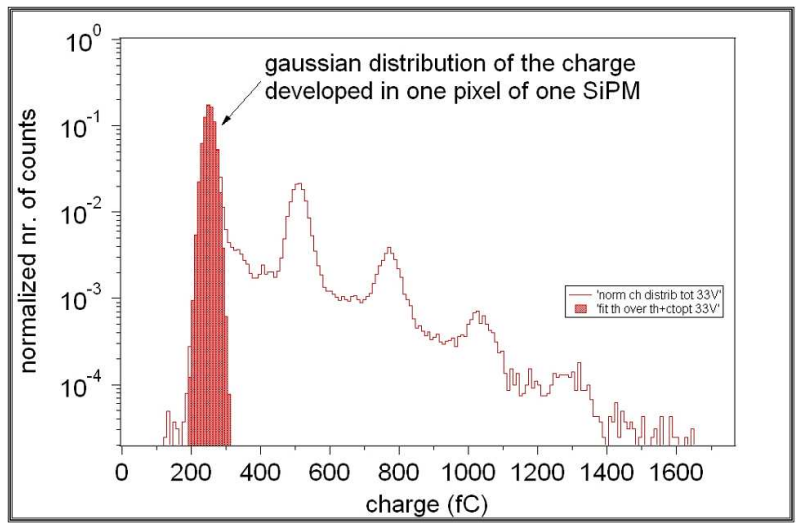

Figure 9. Distribution of the charge obtained with floating integration window.

The DCR shows a direct correlation with the $\mathrm{I}_{\text {post }-B D}$ : the element SiPM13 having the lowest $\mathrm{I}_{\text {post }-B D}$ has the lowest DCR while the element SiPM16 having the highest $\mathrm{I}_{\text {post }-B D}$ has the highest DCR. The remaining 14 SiPM's show a DCR in between the elements SiPM13 and SiPM16, with a mean value $\sim 2 \mathrm{MHz} @ \mathrm{~V}_{\text {bias }}=33 \mathrm{~V}$ and $\sigma \sim 12 \%$ (the same dispersion as for the $\mathrm{I}_{\text {post }-B D}$ ). This suggests that $\mathrm{I}_{\text {post }-B D}$ of a given SiPM represents probably the average current of all contributions (e.g. thermal generated carriers, afterpulses, cross-talk) coming from its pixels.

A spectrum of charges produced by the SiPM was obtained by integration of the output signals shown in figure 7 . If a fixed integration window of $\sim 80 \mathrm{~ns}$ is used (that includes a whole charge of the primary signal), a second pulse (e.g. afterpulse) coming during this integration window is partially integrated. Therefore, a LabView acquisition program using a floating integration window has been developed. In this case, if a primary pulse is followed by a secondary one during its recharge (e.g. $80 \mathrm{~ns}$ ), the integration window (which starts at the primary pulse) is increased with the time interval in between the pulses. Using this method, a complete spectrum of the charge distribution can be obtained (figure 9). Such distribution has a dominant peak (fitted by a Gaussian function) corresponding to primary pulses, followed by a tail corresponding to primary pulses plus secondary effects (afterpulses or cross-talk). 


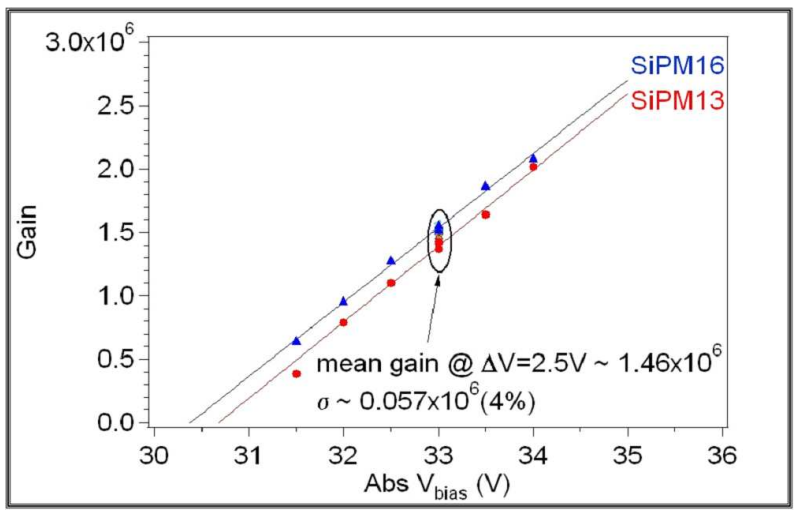

Figure 10. Gain vs. the operation voltage evaluated with the MITEQ amplifier.

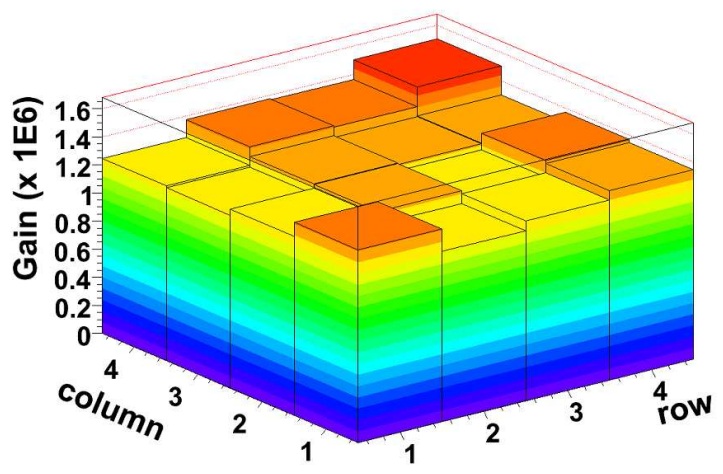

Figure 11. Gain @Vbias=33 V of 16 SiPM's of the matrix.

The centroid of the Gaussian fit represents the most probable charge generated during one primary pulse and it allows determining the SiPM gain as this charge divided by the electron charge. The gain measured using the described method is represented in figure 10 as a function of the $\mathrm{V}_{\text {bias }}$ for the SiPM13 and the SiPM16. A linear increase of the $\mathrm{G}$ with $\mathrm{V}_{\text {bias }}$ has been observed for both elements. The $\mathrm{G}$ has been also evaluated for the other 14 elements of the matrix at $\mathrm{V}_{\text {bias }}$ of $33 \mathrm{~V}$. A mean value of $\sim 1.5 \times 10^{6} @ 33 \mathrm{~V}$ has been determined, with a good uniformity $(\sigma \sim 4 \%)$.

The $\mathrm{G} @ \mathrm{~V}_{\text {bias }}=33 \mathrm{~V}$ for the 16 SiPM's has been also evaluated using the MAROC2 chip. A mean value of $\sim 1.3 \times 10^{6}$ has been determined (figure 11) showing a good agreement in between the $\mathrm{G}$ values measured with both set-ups. The low dispersion $(\sigma \sim 5.5 \%)$ demonstrates not only the gain uniformity through different SiPM's of the matrix but also the uniformity of the channels through the MAROC2 chip.

\subsection{Optical characteristics}

The PDE has been evaluated at low rate and constant flux of incident photons using two methods: the photon counting as well as the photocurrent technique. The first one consists on counting of the signals at the MITEQ amplifier output with particular attention on the threshold value to eliminate 


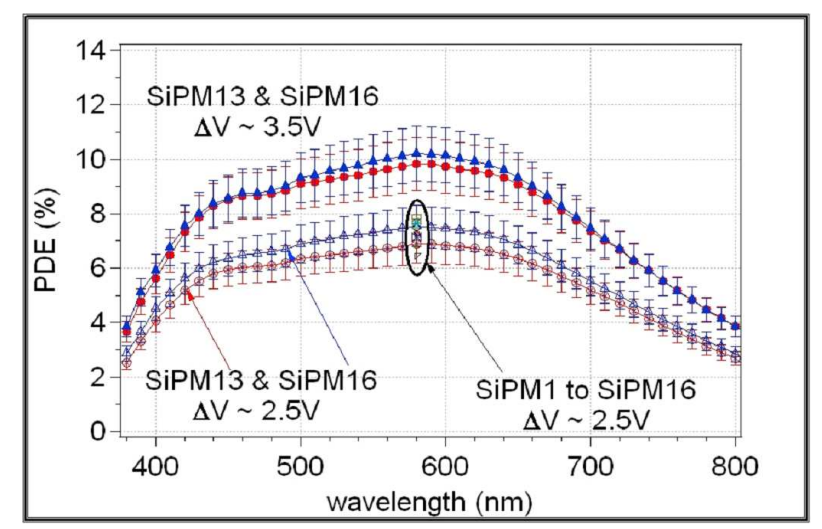

Figure 12. PDE of SiPM13 \& SiPM16 vs. $\lambda$ @ Vbias=33 V and 34 V; PDE of all SiPM's of the matrix @ $\lambda=580 \mathrm{~nm} \&$ Vbias $=33 \mathrm{~V}$.

the afterpulses contribution (only pulses bigger than 0,85 of the primary pulses were counted) and the number of the detected pulses (corrected by the DCR) divided by the number of the incident photons gives the PDE. The second one consists on the measurement of the SiPM photocurrent. The number of detected photons is calculated dividing the photocurrent (corrected by the $\mathrm{I}_{\text {post }-B D}$ ) by the electron charge and the gain. The gain $\mathrm{G}$ reported in figure 10 represents the charge developed only during a primary pulse and it does not take into consideration the charge carried by the afterpulses and cross-talk. Therefore, a corrected gain has been calculated using the weighted average charge over the total spectrum of the charge distribution (figure 9). The PDE is then calculated as the ratio in between the number of the detected photons and the number of incident photons. A good agreement (within an error of $\sim 10 \%$ ) between the two methods has been found.

The PDE as a function of $\lambda$ is represented in figure 12 for the SiPM13 and the SiPM16 at $\mathrm{V}_{\text {bias }}=33 \mathrm{~V}$ and $34 \mathrm{~V}$. The PDE has been also evaluated for the others 14 elements of the matrix at $\mathrm{V}_{\text {bias }}=33 \mathrm{~V}$ and $\lambda=580 \mathrm{~nm}$.

A PDE value of $\sim 8-10 \% @ \mathrm{~V}_{\text {bias }}=34 \mathrm{~V}$ has been found for a wide range of wavelengths $(420-680 \mathrm{~nm})$ as well as a very good uniformity over the elements of the matrix has been observed $(\sigma \sim 5 \%)$.

\section{Conclusions}

In this paper, the electrical and the optical characterization of a prototype matrix of $4 \times 4$ SiPM's of $1 \times 1 \mathrm{~mm}^{2}$ on the same substrat has been presented. The prototype matrix has been produced at FBK-irst and the characterization has been performed at LAL. Both electrical and optical tests performed on the $16 \mathrm{SiPM}$ elements of the matrix proven that the matrix is fully functional and it presents very uniform characteristics. The main features of the matrix are: $\mathrm{V}_{B D} \sim 30.5 \mathrm{~V}$; $\mathrm{R}_{\text {qpixel }} \sim 330 \mathrm{k} \Omega ; \mathrm{I}_{\text {post }-B D} \sim 0.5 \mu \mathrm{A} @ 33 \mathrm{~V}$ directly correlated with the $\mathrm{DCR} \sim 2 \mathrm{MHz} @ 33 \mathrm{~V} ; \mathrm{G} \sim$ $1.5 \times 10^{6} @ 33 \mathrm{~V}$ estimated with two different set-ups; PDE 8-10\%@34 V for a wide range of wavelengths (420-680 nm) estimated by two methods. 


\section{Acknowledgments}

This work is partially supported by the European Commission's $6^{\text {th }}$ Framework Programme through a Marie Curie Intra-European fellowship.

\section{References}

[1] V.M. Golovin, et al., Russian Patent nr. 1644708 (1999).

[2] Z. Sadygov, Russian Patent nr. 2086047 C1 (1997).

[3] P. Buzhan et al., An advances study of silicon photomultiplier, ICFA Instrume. Bull. 23 (2001) 28.

[4] P. Buzhan et al., Large area silicon photomultipliers: Performance and Applications, Nucl. Instrum. Meth. A 567 (2006) 78.

[5] C. Piemonte et al., Recent developments on Silicon Photomultipliers produced at FBK-irst, IEEE Nucl. Sci. Symp. Conf. Rec. 3 (2007) 2089.

[6] G. Llosa et al., First results in the application of Silicon Photomultiplier matrices to small animal PET, in Proceedings of New Developments in Photodetection, June 15-20, 2008, Palais des Congrès, Aix-les-Bains, France, Nucl. Instrum. Meth. A Conference Proceedings.

[7] P. Barrillon et al., MAROC: Multi-Anode ReadOut Chip, Topical Workshop on Electronics for Particle Physics, 3-7 September 2007, Praga Czech Republic.

[8] C. Piemonte et al., Characterization of the first prototypes of Silicon Photomultiplier fabricated at ITC-irst, IEEE Trans. Nucl. Sci. 54 (2007) 236. 